\title{
Multi-Robot Distributed Visual Consensus using Epipoles
}

\author{
Eduardo Montijano
}

Johan Thunberg

\author{
Xiaoming $\mathrm{Hu}$
}

\author{
Carlos Sagues
}

\begin{abstract}
In this paper we give a distributed solution to the problem of making a team of non-holonomic robots achieve the same heading (attitude consensus problem) using vision sensors with limited field of view. The use of cameras with constrained field of view limits the information the robots perceive compared to other omnidirectional sensors. This makes the consensus problem more complicated, because the robots will not always be able to observe other robots. By using structure from motion computed from images, the robots can estimate the difference in their headings from common observations of the environment without the necessity of directly observe each other. In this way, the robots achieve the consensus in their headings while observing the environment instead of each other. The contribution of the paper is a new controller that uses the epipoles computed from pairs of images to estimate the misalignment between neighbor robots. In addition, the control is robust to changes in the topology of the network and does not require to know the calibration of the cameras in order to achieve the desired configuration. To the best of our knowledge, this is the first time that the epipoles are used in multi-robot consensus, putting their properties in value.
\end{abstract}

\section{INTRODUCTION}

The coordination of teams of robots is a problem that has received a lot of attention in the last years. Different approaches solving this problem can be divided into leaderfollower schemes [1]-[5] and nearest neighbor rules [6][10]. In the leader-follower approaches each robot designs its control input considering only the information provided by a single neighbor robot, the leader. In the approaches based on nearest-neighbors rules all the robots play the same role in the formation and each robot designs its control input using the available information provided by direct neighbors in the communication graph. In these approaches there is a natural robustness against changes in the topology and individual failures and for that reason we will focus our research on them.

So far, in the literature, the solutions proposed for the consensus problem using nearest-neighbors rules have focused on the coordination aspects, assuming that the robots measure the orientation of each other, and setting aside the additional interesting task of observing the environment. Solutions that consider omnidirectional range [7] or visual [11] sensors can reach the consensus state while observing the environment without perception problems. However, when the used sensors have field of view constraints, the observation of the neighbors may constrain the perception of the

E. Montijano and C. Sagues are with Departamento de Informática e Ingeniería de Sistemas - Instituto de Investigación en Ingeniería de Aragón (I3A), Universidad de Zaragoza, Spain.

J. Thunberg and X. Hu are with Department of Math, Division of Optimization and Systems Theory, Royal Institute of Technology (KTH), Sweden. environment. While we focus on the problem of making all the robots achieve the same heading (attitude consensus), the solution provided is well suited for perception purposes. At the same time, the attitude consensus can simplify data association or map alignment problems that appear in cooperative perception situations, reducing them to linear translation problems [12].

Our solution assumes that each robot moves on the plane with non-holonomic motion constraints and is equipped with a monocular camera with a limited field of view, pointing forward in the direction of the robot. In order to achieve the consensus in the heading we propose a controller that considers observations of the environment from the images acquired by the robots, using structure from motion (SFM) [13] to compute the misalignment between their headings. Specifically, we use the epipolar constraint to measure the errors in the orientations of the robots. The epipolar constraint is a widely used tool in computer vision and robotic applications due to its natural robustness to mismatching. Although it is possible to extract the relative motion between two images using SFM, we have chosen to use the epipoles in the controller because their computation does not require an explicit decomposition of the fundamental matrix or knowledge about the internal parameters of the camera.

A first approach using the epipoles to control the motion of a robot appeared in [14]. Non-holonomic constraints were introduced in [15], [16]. However, in all the previous approaches the goal is to control one robot and move it to a fixed position, specified by some target image. To the best of our knowledge, this is the first paper in which the epipoles are used for multi-robot control purposes, exploiting their good properties in a distributed coordination application.

The main advantage of our approach is that the robots are not required to directly observe each other while they are still able to align their headings. In addition, our control is fully distributed, independent of the topology of the robotic network and does not require knowledge about the calibration of the cameras in order to achieve the desired configuration.

The rest of the paper is organized as follows: The considered system is described in section II. In section III the vision-based attitude consensus controller is proposed and discussed in detail considering both fixed and switching communication topologies. Experiments in a simulated environment demonstrate the performance of our approach in section IV. Finally, in section V we provide the conclusions. 


\section{DESCRIPTION OF THE SYSTEM}

In this section we introduce the robotic network [17] that describes the system where our controller can be used.

\section{A. Dynamics of the robots}

We consider a homogeneous team of robots moving on the plane. The dynamics of a robot is described by the unicycle model:

$$
\left[\begin{array}{c}
\dot{x}_{i} \\
\dot{z}_{i} \\
\dot{\theta}_{i}
\end{array}\right]=\left[\begin{array}{cc}
\sin \left(\theta_{i}\right) & 0 \\
\cos \left(\theta_{i}\right) & 0 \\
0 & 1
\end{array}\right]\left[\begin{array}{c}
v_{i} \\
w_{i}
\end{array}\right],
$$

where $\left[x_{i}, z_{i}, \theta_{i}\right]^{T} \in \mathbb{R}^{3}$ is the state of robot $i$ (position and orientation) expressed in some world reference frame and $\left[v_{i}, w_{i}\right]^{T} \in \mathbb{R}^{2}$ is the control input of the robot. Along the paper we will consider a constant linear velocity for all the robots, $v_{i}=v \geq 0, \forall i$.

Now, given two robots, $i$ and $j$, we make use of the polar coordinates, distance, $r_{i j}$, bearing angle, $\psi_{i j}$, and relative orientation, $\theta_{i j}$, to describe their relative state

$$
\begin{aligned}
r_{i j} & =\sqrt{x_{i j}^{2}+z_{i j}^{2}} \in \mathbb{R}_{\geq 0}, \\
\psi_{i j} & =\arctan \left(x_{i j} / z_{i j}\right) \in(-\pi / 2, \pi / 2], \\
\theta_{i j} & =\theta_{j}-\theta_{i} \in(-\pi, \pi],
\end{aligned}
$$

where $x_{i j}$ and $z_{i j}$ are the cartesian coordinates of robot $j$ expressed in the reference frame whose origin coincides with robot $i$ (see Fig 1 a).

\section{B. Communications between robots}

Communications between robots are modeled with an undirected graph $\mathcal{G}=(\mathcal{V}, \mathcal{E})$. The nodes in the graph represent the robots, $\mathcal{V}=\{1, \ldots, N\}$ whereas an edge models direct communication between two robots. In this way, if robots $i$ and $j$ are able to exchange messages with each other, then $(i, j) \in \mathcal{E}$. Since communications are undirected $(i, j) \in \mathcal{E} \Leftrightarrow(j, i) \in \mathcal{E}$. The set of neighbors of robot $i$, i.e., the robots that can directly communicate with $i$, is defined as $\mathcal{N}_{i}=\{j \in \mathcal{V} \mid(i, j) \in \mathcal{E}\}$.

We assume that $\mathcal{G}$ is connected, i.e., for every pair of nodes $i$ and $j$, there exists a path of communication links starting in $i$ and ending in $j$. Initially we consider a fixed graph $\mathcal{G}$ over the time and later in the paper we will extend our results to time-varying topologies.

\section{Camera model and output of the system}

In our setup all the robots are equipped with pinhole monocular cameras with limited field of view. For simplicity we will assume that all the robots have identical cameras onboard. The calibration matrix of these cameras is unknown for the robots and equal to $\mathbf{K}=\operatorname{diag}(\alpha, \alpha, 1)$, with $\alpha>0$, being the focal length of the camera measured in pixels. This is equivalent to say that the camera has no skew and that the origin of the image coordinates is fixed on the center of the image. A more detailed treatment about these kind of cameras and their properties can be found, e.g., in [13].

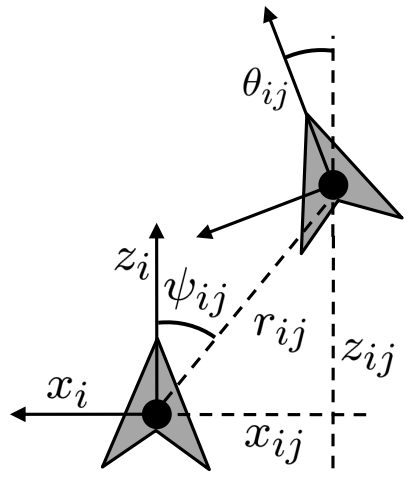

(a)

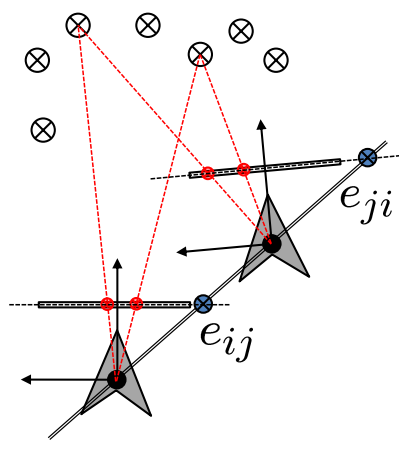

(b)
Fig. 1: (a) Coordinates of robot $j$ in the reference frame of robot $i$. (b) Observation of the neighbor robot with the epipoles. Both robots observe the same features in the scene and using structure from motion they can compute the epipoles without the necessity of observing each other.

The use of monocular cameras implies that the depth of the scene will be unknown to the robots. This means that $r_{i j}$ will not be available to the robots. If the robots are intended to observe the environment, then there will also be many situations in which they will not be able to observe each other in a direct way because of the limited field of view. To overcome this limitation the robots can exchange their images and use structure from motion techniques to estimate their neighbors positions (see Fig. 1 b). However, the lack of knowledge about the calibration of the camera means that the robots will have no direct means to estimate the exact $\psi_{i j}$ and $\theta_{i j}$.

For any pair of neighbor robots, $i$ and $j$, the output of the system will be defined by the epipoles of the images acquired by them. Given a pair of images, it is possible to estimate the fundamental matrix, $\mathbf{F}_{i j}$, that relates them, provided that there are at least 7 correspondences between them [13]. After that, the epipoles, $e_{i j}=\left[e_{i j x}, e_{i j y}\right]^{T}$ and $e_{j i}=\left[e_{j i x}, e_{j i y}\right]^{T}$, can be computed in a linear way as the intersection of the epipolar lines defined by $\mathbf{F}_{i j}$ and the matched features. Due to the planar motion, the y-coordinate of all the epipoles will be equal and constant for any pair of images. The $\mathrm{x}$ coordinate of the epipoles satisfies

$$
e_{i j x}=\alpha \tan \left(\psi_{i j}\right), e_{j i x}=\alpha \tan \left(\psi_{i j}-\theta_{i j}\right) .
$$

For simplicity purposes, in the following we use $e_{i j}$ and $e_{j i}$ to refer to only the $\mathrm{x}$-coordinate of the epipoles.

\section{ATTITUDE SYNCHRONIZATION CONTROLLER}

In the attitude synchronization problem, all the robots in the network shall achieve the same orientation, i.e., $\theta_{i j} \rightarrow$ $0, \forall i, j \in \mathcal{V}$, as $t \rightarrow \infty$. In this section we propose a control law for each robot that uses the epipoles as measurements to achieve this objective.

Given a pair of neighbor robots, by eq. (3), a necessary condition for the attitude alignment is that their epipoles must be equal,

$$
\theta_{i j}=0 \Rightarrow e_{i j}=e_{j i}
$$


However, note that $e_{i j}=e_{j i}$ does not necessarily imply consensus in the orientation because for $\theta_{i j}=\pi$ the two epipoles are also equal. This imposes a natural constraint on the initial orientations of the robots. In order to reach the consensus, we will require that initially $\theta_{i j}<\pi / 2, \forall i, j \in$ $\mathcal{V}$, so that the controller is able to align the robots properly.

We define the misalignment in the epipoles as

$$
w_{i j}= \begin{cases}d_{i j} & \text { if }\left|d_{i j}\right| \leq \frac{\pi}{2} \\ -\operatorname{sign}\left(d_{i j}\right)\left(\pi-\left|d_{i j}\right|\right) & \text { otherwise }\end{cases}
$$

where

$$
d_{i j}=\arctan \left(\frac{e_{i j}}{\beta}\right)-\arctan \left(\frac{e_{j i}}{\beta}\right) \in(-\pi, \pi],
$$

and $0<\beta<\infty$ is some fixed positive constant to choose.

Several aspects justify this misalignment function. First of all, eq. (5) is a bijective mapping $(-\infty, \infty) \rightarrow(-\pi, \pi)$ that reduces the misalignment in the epipoles to quantities that represent something more similar to angular distances. This reduction also implies smaller control gains. Secondly, equation (4) introduces the geodesic distance in the difference between the epipoles and is used to select the closest path (clockwise or anti-clockwise) that makes both epipoles be the same. Finally, note that, if $\beta=\alpha$, then the setup is calibrated, $d_{i j}=\theta_{i j}$, and the relative orientation between the robots can be computed from the epipoles. However, for the moment we assume that this is not the case and $\beta \neq \alpha$.

Since the robots can have more than one neighbor, the control input $w_{i}$ of each robot is defined as:

$$
w_{i}=K \sum_{j \in \mathcal{N}_{i}} w_{i j}
$$

where $K>0$ is the controller gain.

In order to prove the stability of the proposed controller, we will make use of the following lemma.

Lemma 3.1: The control defined in (4) and (6) satisfies the following properties:

1) $w_{i j}=-w_{j i}$.

2) $\sum_{i \in \mathcal{V}} w_{i}=0$

3) $\operatorname{sign}\left(e_{i j}\right)=\operatorname{sign}\left(e_{j i}\right) \Rightarrow\left|d_{i j}\right|<\pi / 2$.

Proof: First note that $d_{i j}=-d_{j i}$. Therefore, if $\left|d_{i j}\right| \leq$ $\pi / 2$, then $w_{i j}=-w_{j i}$. In eq. (4), when $\left|d_{i j}\right|>\pi / 2,(\pi-$ $\left.\left|d_{i j}\right|\right)$ has the same sign that $\left(\pi-\left|d_{j i}\right|\right)$ because $\left|d_{i j}\right| \leq \pi$. But $\operatorname{sign}\left(d_{i j}\right) \neq \operatorname{sign}\left(d_{j i}\right)$, which implies that $w_{i j}=-w_{j i}$ and 1) is proved.

The proof of 2) is done decomposing the sum of $w_{i}$,

$$
\sum_{i \in \mathcal{V}} w_{i}=K \sum_{(i, j) \in \mathcal{E}} w_{i j}
$$

Taking into account that the communication graph is undirected and $w_{i j}=-w_{j i}$, then the sum is equal to zero.

To prove 3) let us consider that both epipoles have the same sign, without loss of generality, positive. The arc tangents have values in the interval $[0, \pi / 2)$ and therefore, the difference in eq. (5) belongs to the interval $(-\pi / 2, \pi / 2)$.
Theorem 3.2: Consider a robotic network like the one defined in section II, with the robots initially oriented in such a way that $\left|\theta_{i j}\right| \leq \theta_{M}<\pi / 2, \forall i, j \in \mathcal{V}$. If the robots use the control law (6) with $\beta$ satisfying

$$
\alpha \tan \left(\frac{\theta_{M}}{2}\right)<\beta<\frac{\alpha}{\tan \left(\frac{\theta_{M}}{2}\right)},
$$

then $\lim _{t \rightarrow \infty} \theta_{i j}=0, \forall i, j \in \mathcal{V}$, i.e., the system will reach consensus.

Proof: Let $\boldsymbol{\theta}(t)=\left(\theta_{1}(t), \ldots, \theta_{N}(t)\right)$. The proof is done using the following Lyapunov function

$$
V(\boldsymbol{\theta})=\sum_{i \in \mathcal{V}} \sum_{j \in \mathcal{V}} \frac{1}{2}\left(\theta_{j}-\theta_{i}\right)^{2}=\sum_{i \in \mathcal{V}} \sum_{j \in \mathcal{V}} \frac{1}{2} \theta_{i j}^{2} \geq 0 .
$$

Note that due to the connectivity assumption, (8) is positive definite in terms of, for example, $\theta_{i}-\theta_{1}, i=2, \ldots, N$. If we compute the derivative of $V$ we obtain

$$
\dot{V}=\sum_{i \in \mathcal{V}} \sum_{j \in \mathcal{V}}\left(\theta_{j}-\theta_{i}\right)\left(w_{j}-w_{i}\right)
$$

We proceed to show that the derivative is negative if $\theta_{i j} \neq 0$. First, by developing (9) we obtain

$$
\dot{V}=2 N \sum_{i \in \mathcal{V}} \theta_{i} w_{i}-\sum_{i \in \mathcal{V}} \theta_{i} \sum_{j \in \mathcal{V}} w_{j}-\sum_{i \in \mathcal{V}} w_{i} \sum_{j \in \mathcal{V}} \theta_{j},
$$

which by the second point of Lemma 3.1 is simplified to

$$
\dot{V}=2 N \sum_{i \in \mathcal{V}} \theta_{i} w_{i}
$$

Now, regrouping the terms yields

$$
\sum_{i \in \mathcal{V}} \theta_{i} w_{i}=K \sum_{i \in \mathcal{V}} \theta_{i} \sum_{j \in \mathcal{N}_{i}} w_{i j}=\frac{K}{2} \sum_{(i, j) \in \mathcal{E}}\left(\theta_{i}-\theta_{j}\right) w_{i j}
$$

Therefore, the derivative of $V$ can be expressed as

$$
\dot{V}=-K N \sum_{(i, j) \in \mathcal{E}} \theta_{i j} w_{i j}
$$

We show now that under the conditions stated in the theorem, the product $\theta_{i j} w_{i j}$ is positive for all $i, j$. Let us first suppose that $\theta_{i j}>0$. We divide the analysis in four cases. The first two cases consider positive bearing angles:

- Let $\psi_{i j}$ be positive and satisfying $\psi_{i j} \geq \theta_{i j}$. In this case $e_{i j}>e_{j i} \geq 0$. Since both epipoles have the same sign, using the third point of Lemma 3.1, $0<d_{i j}<\pi / 2$ and then $w_{i j}>0$. Note that this case does not depend on the selection of $\beta$, provided that it has the same sign as $\alpha$.

- If $\theta_{i j}>\psi_{i j} \geq 0$ then $e_{j i}<0<e_{i j}$, which implies that $d_{i j} \geq 0$. However, if $d_{i j}>\pi / 2$, then $w_{i j}<0$ and the control may not be stable. In order to have $w_{i j}>0$ it must hold that $d_{i j} \leq \pi / 2$, which is equivalent as to say that $\tan \left(d_{i j}\right)>0$, therefore, using (3) and (5),

$$
\tan \left(d_{i j}\right)=\frac{\frac{\alpha}{\beta}\left(\tan \left(\psi_{i j}\right)-\tan \left(\psi_{i j}-\theta_{i j}\right)\right)}{1+\frac{\alpha^{2}}{\beta^{2}} \tan \left(\psi_{i j}\right) \tan \left(\psi_{i j}-\theta_{i j}\right)}>0 .
$$


The numerator in (11) is always positive due to the conditions on $\theta_{i j}$ and $\psi_{i j}$. Then, to satisfy (11) it is required that

$$
1+\frac{\alpha^{2}}{\beta^{2}} \tan \left(\psi_{i j}\right) \tan \left(\psi_{i j}-\theta_{i j}\right)>0
$$

thus

$$
\frac{\alpha}{\beta}<\sqrt{\frac{1}{\tan \left(\psi_{i j}\right) \tan \left(\theta_{i j}-\psi_{i j}\right)}},
$$

which depends on the ratio $\alpha / \beta$. A lower bound of the right side of eq. (12) is provided later in the proof.

Let us now analyze the cases of negative bearing angles:

- Let us consider first $\psi_{i j}<0$ and $\theta_{i j}-\psi_{i j}<\pi / 2$. When this situation happens $e_{j i}<e_{i j}<0$ and $w_{i j}>0$ because of the third point of Lemma 3.1. Again, when the robots are in this configuration, the control does not depend on $\beta$.

- The last case to analyze appears when $\psi_{i j}<0$ and $\theta_{i j}-\psi_{i j}>\pi / 2$. In this situation the epipoles have different sign, with $e_{i j}<0<e_{j i}$, which implies that, in order to have $w_{i j}>0$, it must happen that $d_{i j}<-\pi / 2$. In other words, $\tan \left(d_{i j}\right)>0$. Now, the numerator in (11) is always negative, which requires

$$
1+\frac{\alpha^{2}}{\beta^{2}} \tan \left(\psi_{i j}\right) \tan \left(\psi_{i j}-\theta_{i j}\right)<0,
$$

in order to fulfill (11), and then

$$
\frac{\alpha}{\beta}>\sqrt{\frac{1}{\tan \left(\psi_{i j}\right) \tan \left(\theta_{i j}-\psi_{i j}\right)}} .
$$

Note that (12) and (13) are not in conflict because they are evaluated in different ranges of $\psi_{i j}$. An upper bound of the right side of eq. (13) is provided later in the proof.

The analysis when $\theta_{i j}<0$ can be done taking into account the first point of Lemma 3.1. Using eq. (2), $-\theta_{i j}=$ $\theta_{j i}>0$, then $w_{j i}>0$ and $w_{i j}<0$. The system is in equilibrium when $\theta_{i j}=0, \forall(i, j) \in \mathcal{E}$, but due to the fact that the communication graph is connected, then the set of equilibrium points is $\theta_{i j}=0, \forall i, j \in \mathcal{V}$.

We compute now the bounds that satisfy (12) and (13). Let

$$
\gamma\left(\theta_{i j}, \psi_{i j}\right)=\sqrt{\frac{1}{\tan \left(\psi_{i j}\right) \tan \left(\theta_{i j}-\psi_{i j}\right)}} .
$$

We analyze (14) in the intervals $\mathcal{I}_{1}$ and $\mathcal{I}_{2}$

$$
\begin{aligned}
& \mathcal{I}_{1}=\left\{\left(\theta_{i j}, \psi_{i j}\right) \mid 0<\psi_{i j}<\theta_{i j}<\theta_{M}\right\}, \\
& \mathcal{I}_{2}=\left\{\left(\theta_{i j}, \psi_{i j}\right) \mid \psi_{i j}<0<\theta_{i j}<\theta_{M}, \psi_{i j}-\theta_{i j}<-\frac{\pi}{2}\right\},
\end{aligned}
$$

The partial derivative of (14) with respect to $\theta_{i j}$ is equal to

$$
\frac{\partial \gamma}{\partial \theta_{i j}}=\frac{-1}{2 \gamma \tan \left(\psi_{i j}\right) \tan ^{2}\left(\theta_{i j}-\psi_{i j}\right) \cos ^{2}\left(\psi_{i j}-\theta_{i j}\right)} .
$$

We can see that the sign of (15) depends only on the sign of $\tan \left(\psi_{i j}\right)$, which is positive on $\mathcal{I}_{1}$ and negative on $\mathcal{I}_{2}$. Therefore, the function is decreasing with $\theta_{i j}$ on $\mathcal{I}_{1}$ and increasing on $\mathcal{I}_{2}$ and in both cases the bound we are looking for will be achieved in $\theta_{i j}=\theta_{M}$.

If we compute the derivative of (14) with respect to $\psi_{i j}$, already considering $\theta_{i j}=\theta_{M}$ we obtain

$$
\frac{\partial \gamma}{\partial \psi_{i j}}=\frac{\sin \left(\theta_{M}-\psi_{i j}\right) \cos \left(\theta_{M}-\psi_{i j}\right)-\sin \left(\psi_{i j}\right) \cos \left(\psi_{i j}\right)}{2 \gamma \sin ^{2}\left(\theta_{M}-\psi_{i j}\right) \sin ^{2}\left(\psi_{i j}\right)} .
$$

The only minimum of (16) on the interval $\mathcal{I}_{1}$ is in $\psi_{i j}=$ $\theta_{M} / 2$. The maximum on $\mathcal{I}_{2}$ is found on the value $\psi_{i j}=$ $-\pi / 2+\theta_{M} / 2$. Using trigonometry equivalences we obtain that

$$
\gamma\left(\theta_{M},-\pi / 2+\theta_{M} / 2\right)=\frac{1}{\gamma\left(\theta_{M}, \theta_{M} / 2\right)} .
$$

Finally, by noting that $\gamma\left(\theta_{M}, \theta_{M} / 2\right)=1 / \tan \left(\theta_{M} / 2\right)$, the condition in (7) is obtained.

The last point to check is the invariance of the set $\left|\theta_{i j}\right| \leq$ $\theta_{M}, \forall i, j$. To show this, let us consider a fixed reference frame $\mathcal{F}$, and let $\theta_{\max }$ and $\theta_{\min }$ be the maximum and minimum orientation values in such frame. This means that, initially, $\max \theta_{i j}=\theta_{\max }-\theta_{\min } \leq \theta_{M}$ and $\theta_{i} \in\left[\theta_{\min }, \theta_{\max }\right]$, for all $i$. Now, let us note that $\theta_{\max i}=\theta_{i}-\theta_{\max } \leq 0$, and $\theta_{\min i}=\theta_{i}-\theta_{\min } \geq 0$ for all $i$ and all $t$. Therefore, $w_{\max } \leq 0$ and $w_{\min } \geq 0$. Since the orientations are in a manifold, it is possible that $w_{\max } \leq-\pi$ and even when it has negative sign the difference $\theta_{\max }-\theta_{\min }$ is increased. By choosing $K$ sufficiently small, e.g., such that $w_{\max }>-\theta_{M}$ and $w_{\min }<\theta_{M}$, we can guarantee that the extremes of the set are always pushed to the interior, proving its invariance.

Besides the stability of the system, the theorem provides a relation between the calibration parameter and the relative orientation between the agents. We did not consider in the proof the special cases $\psi_{i j}=0, \psi_{i j}=\pi / 2, \psi_{i j}=\theta_{i j}$ and $\psi_{i j}-\theta_{i j}= \pm \pi / 2$ to compute the bounds (12) and (13). However, it can be shown that the controller (6) is always well defined in these situations independently of $\beta$. Now we proceed to show the behavior of the controller when the topology of the communications changes over the time.

\section{A. Analysis Under Switching Topologies}

In our system, we should take into account that the motion in the robots may introduce some changes in the graph that defines the communication topology. Perception issues also arise in this context that may affect the neighborhood of each robot. It is possible that two neighboring robots cannot compute their epipoles due to blurry images or temporal occlusions, which would be the same as to assume that they are not neighbors in the communication graph. Finally, computational issues should also be considered to model the communications using a time-varying graph. The computation of the epipoles using a robust algorithm, e.g., DLT+RANSAC [13], requires some time and, although one or two fundamental matrices can be computed in a reasonable amount of time, robots with a larger number of neighbors may not be able to keep up with the rhythm of the continuous time controller. 

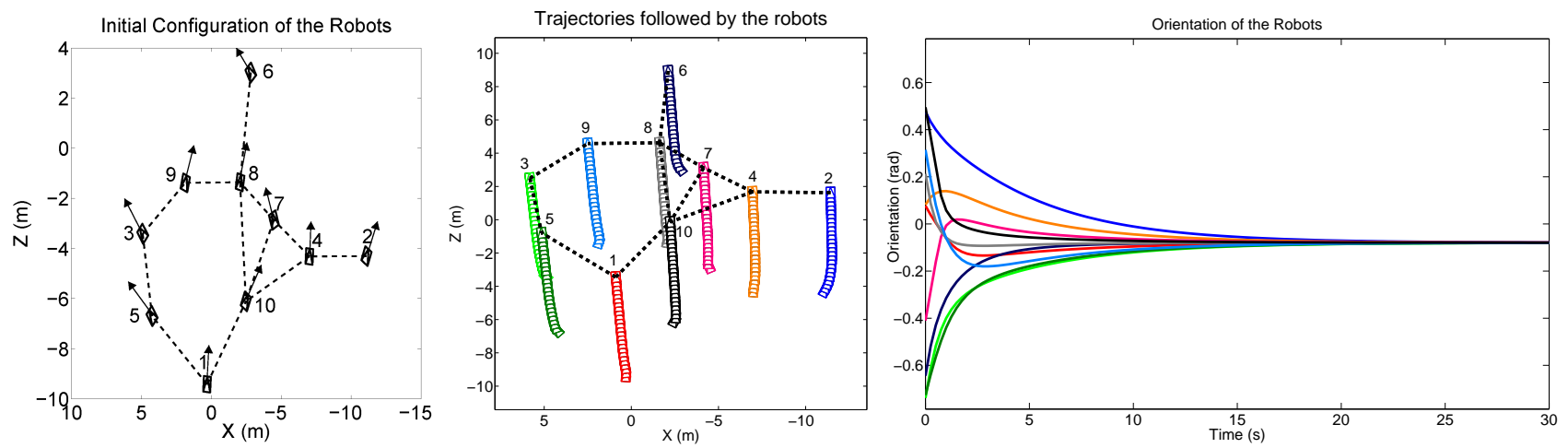

Fig. 2: Initial positions (left) and trajectories followed by the robots (middle). Dashed lines represent direct communications between robots. In the right figure we can observe the values of the orientation of the robots, which eventually reach the consensus.

For all these reasons it is interesting to analyze the controller in the presence of changes in the communication topology. In this paper we show the convergence of the controller, leaving for future work the selection of the links to reduce the number of SFM computations while preserving connectivity along the time.

Before stating the convergence result we impose two standard assumptions when considering switching topologies.

Assumption 3.1: There exists a lower bound, $\delta>0$, on the time between two consecutive changes in the topology. Denoting $t_{k}, k \in \mathbb{N}$, the discrete time instants when the topology changes, then $t_{k+1}-t_{k} \geq \delta, \forall k$.

Assumption 3.2: There exists a positive time period $T$ such that, for any instant of time, $t$, the collection of communication topologies in the time interval $(t, t+T)$ is jointly connected.

Proposition 3.3: Consider a robotic network like the one defined in section II, which satisfies the conditions stated in Theorem 3.2 and Assumptions 3.1 and 3.2. If the robots use the control law

$$
w_{i}=K \sum_{j \in \mathcal{N}_{i}(t)} w_{i j}
$$

then $\lim _{t \rightarrow \infty} \theta_{i j}=0, \forall i, j \in \mathcal{V}$.

The proof is very similar to the standard case where the robots perceive each other heading, e.g., [8], and for brevity is omitted in the paper.

\section{Simulations}

In this section, the properties of the proposed controller are shown in simulations. The experiments have been carried out using Matlab. We have considered a robotic network composed by ten robots with initial positions and orientations depicted in Fig. 2 and communications defined by the dashed lines. To simulate the vision system we have randomly generated a set of $3 \mathrm{D}$ features in the environment. The cameras have calibration matrix $\mathbf{K}=\operatorname{diag}(300,300,1)$, and a resolution of $640 \times 480$ pixels. This implies that the robots have a limited field of view of 94 degrees. Under these conditions not all the robots can observe each other in their images. For example, robot two can only communicate with robot four and there are no other robots visible in its field of view. However, using the epipoles it can compute a control input to align its heading with the one of robot four.
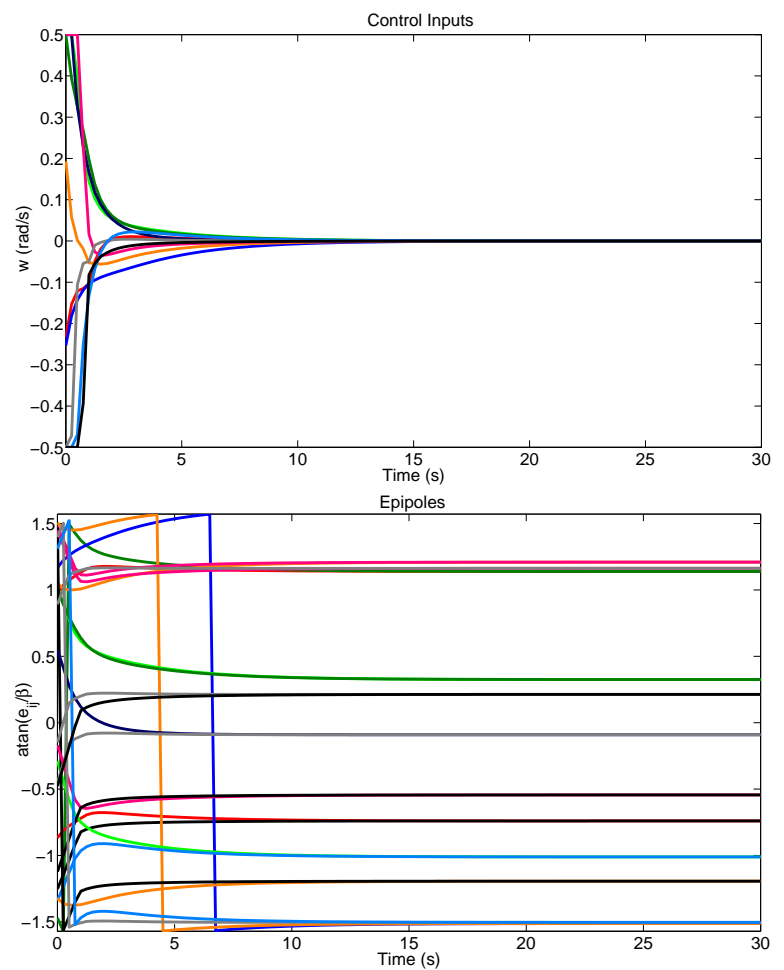

Fig. 3: Control inputs and evolution of the pairs of computed epipoles for the robotic network in Fig. 2.

The results of using the proposed controller with $\beta=250$ are shown in Figs. 2 and 3. Since the maximum relative orientation between a pair of robots is 1.23 the bounds on $\beta$ required to converge are $212<\beta<423$ and in this case the controller reaches the consensus. The right figure in Fig. 2 shows the evolution of the orientation of the robots, which converge to the same value for all of them. The top figure in Fig. 3 depicts the control inputs and the bottom figure the evolution of all the pairs of computed epipoles.

\section{A. Example with switching topologies}

We have also introduced in the simulation some constraints to make it more realistic. To consider the time required for the computation of the epipoles we have discretized the controller with time step equal to 0.25 seconds. At each iteration, each robot randomly selects a subset of its neighbors to compute the epipoles. This selection generates 

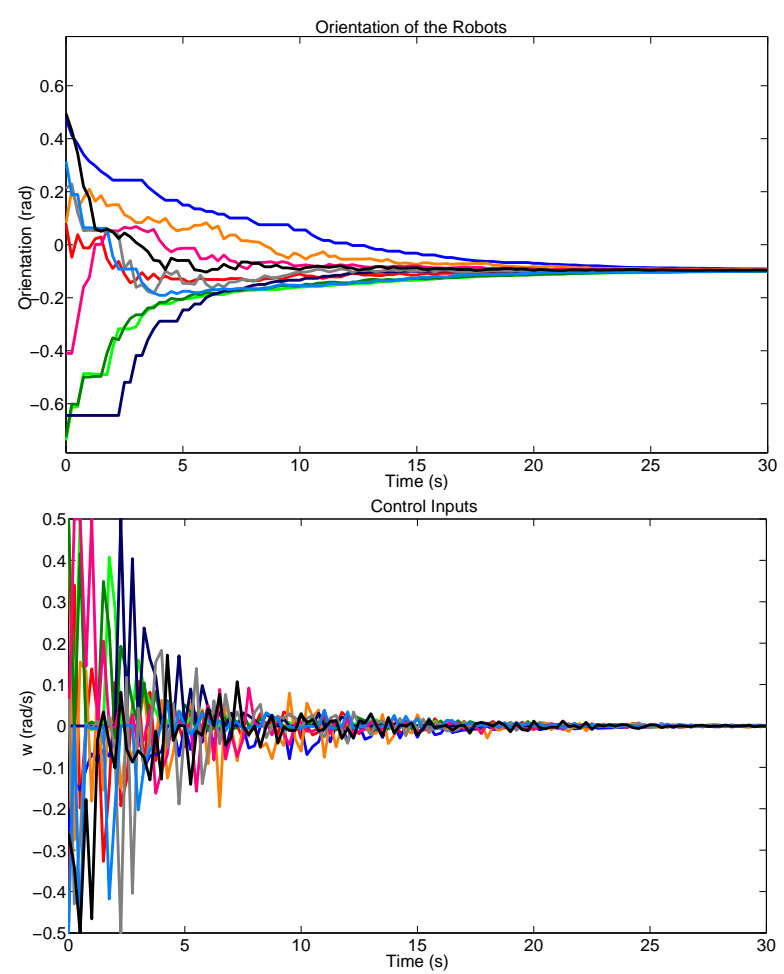

Fig. 4: Evolution of the network with switching topologies. Orientation of the robots (top) and control inputs (bottom).

changes in the network topology, transforming the system into a switching one. Also this selection reduces the number of fundamental matrices that the robots need to compute, improving the computational cost. The dwell time required in Assumption 3.1 comes from the discretization. Joint connectivity is preserved because along the time all the links of the original graph (which is connected) are selected at some point. The results of the evolution of the system are shown in Fig 4. We can see that the robots still achieve the attitude alignment.

\section{Conclusions}

In this paper we have presented a new vision-based distributed controller that allows a team of robots to achieve a common heading while they observe the environment. The proposed controller makes use of the epipoles computed between the images of neighboring robots to estimate the misalignment in their orientations. The use of the epipoles presents several advantages: they do not require explicit computation of the relative motion between the robots or knowledge about the calibration of the cameras and there are well known robust techniques for their computation. We have provided the conditions required for the controller such that the system will reach consensus. Several simulations show the behavior of the approach in different situations. Our future work will focus on proposing an intelligent link selection to reduce the computational costs derived of the vision system and on extending the controller with selfcalibration tools that will allow the robots to use different cameras or more complex calibration models.

\section{ACKNOWLEDGMENTS}

This work was supported by the project DPI2009-08126 and grant AP2007-03282 Ministerio de Educacion y Ciencia and partly supported by Swedish Research Council and Swedish Foundation for Strategic Research.

\section{REFERENCES}

[1] A. Das, R. Fierro, V. Kumar, J. Ostrowski, J. Spletzer, and C. J. Taylor. Vision based formation control of multiple robots. IEEE Transactions on Robotics and Automation, 18(5):813-825, Oct 2002.

[2] T. Gustavi and X. Hu. Observer-based leader-following formation control using onboard sensor information. IEEE Transactions on Robotics, 24(6):1457-1462, Dec 2008.

[3] J. A. Marshall, M. E. Broucke, and B. A. Francis. Pursuit formations of unicycles. Automatica, 42(1):3-12, 2006.

[4] P. Urcola and L. Montano. Cooperative robot team navigation strategies based on an environment model. In IEEE/RSJ International Conference on Intelligent Robots and Systems, pages 4577-4583, St. Louis, USA, October 2009.

[5] T. Ibuki, T. Hatanaka, M. Fujita, and M. W. Spong. Visual feedback attitude synchronization in leader-follower type visibility structures. In 49th IEEE Conference on Decision and Control, pages 2486-2491, December 2010.

[6] M. Basiri, A. N. Bishop, and P. Jensfelt. Distributed control of triangular formations with angle-only constraints. Systems and Control Letters, 59(2):147-154, 2010.

[7] A. Jadbabaie, J. Lin, and A. S. Morse. Coordination of groups of mobile autonomous agents using nearest neighbor rules. IEEE Transactions on Automatic Control, 48(6):988-1001, June 2003.

[8] N. Mostagh and A. Jadbabaie. Distributed geodesic control laws for flocking of nonholonomic agents. IEEE Transactions on Automatic Control, 52(4):681-686, 2007.

[9] A. Ganguli, J. Cortes, and F. Bullo. Multirobot rendezvous with visibility sensors in nonconvex environments. IEEE Transactions on Robotics, 25(2), 2009.

[10] J. Cortés, S. Martínez, T. Karatas, and F. Bullo. Coverage control for mobile sensing networks. IEEE Transactions on Robotics and Automation, 20(2):243-255, 2004.

[11] N. Mostagh, N. Michael, A. Jadbabaie, and K. Daniilidis. Visionbased, distributed control laws for motion coordination of nonholonomic robots. IEEE Transactions on Robotics, 25(4):851-860, 2009.

[12] J. Cortes. Global and robust formation-shape stabilization of relative sensing networks. Automatica, 45(12):2754 - 2762, 2009.

[13] Y. Ma, S. Soatto, J. Kosecka, and S. S. Sastry. An Invitation to $3 D$ Vision. SpringerVerlag, 2004.

[14] R. Basri, E. Rivlin, and I. Shimshoni. Visual homing: Surfing on the epipoles. International Journal of Computer Vision, 33(2):117-137, 1999.

[15] G. López-Nicolás, C. Sagues, J.J. Guerrero, D. Kragic, and P. Jensfelt. Switching visual control based on epipoles for mobile robots. Robotics and Autonomous Systems, 56(7):592-603, 2008.

[16] G.L. Mariottini, G. Oriolo, and D. Prattichizzo. Image-based visual servoing for nonholonomic mobile robots using epipolar geometry. IEEE Transactions on Robotics, 23(1):87-100, February 2007.

[17] F. Bullo, J. Cortés, and S. Martínez. Distributed Control of Robotic Networks. Applied Mathematics Series. Princeton University Press, 2009. Electronically available at http://coordinationbook.info. 